\title{
A class of line-transformed cloaks with easily-realizable constitutive parameters
}

\author{
Wei Xiang Jiang, Hui Feng Ma, Qiang Cheng and Tie Jun Cui* \\ State Key Laboratory of Millimeter Waves and Institute of Target Characteristics and Identification, \\ Department of Radio Engineering, Southeast University, Nanjing 210096, P. R. China.
}

\begin{abstract}
We propose a class of line-transformed cylindrical cloaks which have easily-realizable constitutive parameters. The scattering properties of such cloaks have been investigated numerically for both transverseelectric (TE) and transverse-magnetic (TM) incidences of plane waves. A line-transformed invisibility cloak with a perfectly electric conducting (PEC) inner boundary is actually a reshaping of a PEC line to which the cloaked object is crushed. The numerical results of near-field distributions and far-field scattering properties have verified the above conclusions. We also investigate the relationship between the constitutive parameters of a line-transformed cloak and the length of the corresponding line. The changing range of constitutive parameters is large when the line is short, while the changing range becomes small when the line is long. The above conclusion provides an efficient way to realize the invisibility cloaks using artificial metamaterials.
\end{abstract}

Key words: Metamaterial, line-transformed invisibility cloak, optical transformation.

PACS numbers: 41.20.Jb, 42.25.Gy, 42.79.-e

*E-mail: tjcui@seu.edu.cn 


\section{Introduction}

In the past three years, great attention has been paid to the invisibility cloaks due to their fascinating and exciting properties [1-23]. The interesting idea to control the electromagnetic (EM) waves was proposed in Refs. [1] and [2]. The first free-space cloak was experimentally demonstrated at the microwave frequency using the reduced constitutive parameters [3]. Later, Li and Pendry have proposed a kind of ground-plane cloak recently [4], which was verified soon in the microwave regime [5] and in the optical regime [6,7]. Many further analytical and numerical investigations have also been devoted in the recently years [8-23].

To design a free-space cloak, one usually transforms an enclosed space to an annular space with the outer boundary unchanged [1]. As illustrated in Fig. 1(c), the region enclosed by boundary $s$ is compressed to the annular region bounded by the outer boundary $s$ and an inner boundary $s_{0}$. Such a space mapping can be expressed as a specific coordinate transformation. Generally, the inner boundary $s_{0}$ is obtained by blowing up a line or a point as shown in Figs. 1(a) and 1(b) [23]. Thus the invisibility cloaks can be divided into two classes: point-transformed cloaks and line-transformed cloaks. The green region in Fig. 1(c) is the desired cloaking layer.

One problem for the point-transformed full-parameter cloaks is that the parameters always exist singularities on the inner boundary. In other words, some parameter components approaches infinity near the inner boundary, which is difficult to reach even using modern metamaterials [3]. Recently, the circular cloaks $[14,16]$, elliptical cloaks [17-20], and other-shaped cloaks [13,21,23] have been designed and studied in different coordinate systems. However, the material parameters for the above cloaks still have infinite values on the inner boundary. Mathematically, such cloaks try to crush an shielded region to a point, which leads to the singularity of constitutive parameters.

In view of the difficulty to realize the point-transformed full-parameter cloak, a ground-plane cloak which can hide the object under a metamaterial carpet has been proposed [4]. Unlike the completely free-space cloaks, the ground-plane cloak crushes the hidden object to a conducting wire. Suck a carpet-like cloak does not require extreme values of the material parameters. But the carpet-like cloak can only shield object under a conducting surface, and cannot shield objects in free space.

In this work, we propose a class of free-space line-transformed cylindrical cloaks which have easilyrealizable constitutive parameters. Instead of shrinking the concealed object to a point as usual, such cloaks crush the object to a line segment. The scattering properties of such cloaks have been investigated numerically for both transverse-electric (TE) and transverse-magnetic (TM) incidences of plane waves. A line-transformed invisibility cloak with a perfectly electric conducting (PEC) inner boundary is indeed a 
reshaper of a PEC line to which the cloaked object is crushed. Numerical results of near-field distributions and scattering patterns have verified the above conclusions. We also investigate the relationship between the constitutive parameters of a line-transformed cloak and the length of the corresponding line. The changing range of constitutive parameters is large when the line is short, while the changing range becomes small when the line is long. The proposed invisibility cloaks could be realized using artificial metamaterials.

\section{Line-transformed cloaks and constitutive parameters}

Using the unique feature of classical elliptically-cylindrical coordinate system, we construct a class of linetransformed cloaks. The relationship between this coordinate system $(\xi, \eta, z)$ and the Cartesian coordinates $(x, y, z)$ is written as

$$
x=p \cosh \xi \cos \eta, \quad y=p \sinh \xi \sin \eta, \quad z=z,
$$

in which $p$ is the half focus of the ellipse. We note that in this coordinate system, if $p$ is assumed to be constant, then isolines for $\xi$ can be a series of elliptical cylindrical shells with the same focus value. In particular, $\xi=0$ means a line segment $(y=0, p \leq x \leq p)$ with length $2 p$. To construct a line-transformed cloak, a general spatial transformation from the elliptical region $\xi \in\left[0, \xi_{2}\right]$ to the annular region $\xi^{\prime} \in\left[\xi_{1}, \xi_{2}\right]$ can be expressed as follows

$$
\xi^{\prime}=f(\xi), \eta^{\prime}=\eta, z^{\prime}=z
$$

in which $f$ is a continuous and differentiable function with $f(0)=a_{1}$ and $f\left(a_{2}\right)=a_{2}$, where $a_{1}$ and $a_{2}$ are coordinates of the inner and outer boundaries of the cloak. The relationship between coordinates and lengths of major axes can be expressed as $a_{1}=\ln \left(r_{1} / p+\sqrt{\left(r_{1} / p\right)^{2}-1}\right)$ and $a_{2}=\ln \left(r_{2} / p+\sqrt{\left(r_{2} / p\right)^{2}-1}\right)$, where $r_{1}$ and $r_{2}$ are the lengths of major axes for inner and outer shells of the cloak. Obviously, the inner boundary is crushed to the line segment $2 p$ using the above coordinate transformation.

The constitutive tensors of the cloak in an arbitrary coordinate system are given as the following equations

$$
\overline{\varepsilon^{\prime}}=\Lambda \bar{\varepsilon} \Lambda^{T} / \operatorname{det}(\Lambda), \quad \overline{\mu^{\prime}}=\Lambda \bar{\mu} \Lambda^{T} / \operatorname{det}(\Lambda) .
$$

Here, the Jacobian matrix is

$$
\Lambda=\left(\begin{array}{ccc}
f^{\prime} g & 0 & 0 \\
0 & g & 0 \\
0 & 0 & 1
\end{array}\right),
$$


in which $f^{\prime}$ is the derivative of $f$ with respect to $\xi$ and $g=\sqrt{\cosh 2 \xi^{\prime}-\cos 2 \eta} / \sqrt{\cosh 2 \xi-\cos 2 \eta}$. The invisibility cloak is considered to be placed in the free space, in other words, $\bar{\varepsilon}=\varepsilon_{0} \bar{I}, \bar{\mu}=\mu_{0} \bar{I}$. Then the relative permittivity and permeability tensors of the line-transformed cloak are expressed as

$$
\begin{aligned}
& \varepsilon_{\xi^{\prime}}^{\prime}=\mu_{\xi^{\prime}}^{\prime}=f^{\prime} \\
& \varepsilon_{\eta^{\prime}}^{\prime}=\mu_{\eta^{\prime}}^{\prime}=\frac{1}{f^{\prime}} \\
& \varepsilon_{z^{\prime}}^{\prime}=\mu_{z^{\prime}}^{\prime}=\frac{1}{f^{\prime}} \frac{\cosh 2 f^{-1}-\cos 2 \eta^{\prime}}{\cosh 2 \xi^{\prime}-\cos 2 \eta^{\prime}}
\end{aligned}
$$

where $f^{-1}$ denotes the inverse function of $f$. The cloak is considered to be lossless in the working frequency. For the sake of simplicity and real applications, we choose $f$ as a linear function $f(\xi)=\left(a_{2}-a_{1}\right) \xi / a_{2}+a_{1}$ in the following discussions. As a result, we obtain $f^{\prime}=\left(a_{2}-a_{1}\right) / a_{2}$ and $f^{-1}\left(\xi^{\prime}\right)=a_{2}\left(\xi^{\prime}-a_{1}\right) /\left(a_{2}-a_{1}\right)$.

Equations (4)-(6) provide full-design material parameters for a general line-transformed cloak in the classical elliptical-cylindrical coordinates. The relationship between the permittivity and permeability components and the length of the line segment $2 p$ has been shown in Figure 2, in which we choose the lengths of major axes as $r_{1}=0.2 \mathrm{~m}$ and $r_{2}=0.4 \mathrm{~m}$. To visualize the changing ranges of material parameters in cloaking layer better, we plot the parameter components along the line $\eta^{\prime}=0$. When the half focus $p$ changes from 0 to $r_{1}$, the $\xi$ and $\eta$ components of the material parameters are shown in Fig. 2(a), and the $z$ component ranges from 0 to $\eta$ as shown in Fig. 2(b), which can be easily observed from Eq. (6). Clearly, this set of material parameters have no singularities because the line-transformed cloak crushes the concealed object to a line segment with nonzero length $2 p$.

For practical reasons, we choose two cases $p=0.0476 \mathrm{~m}$ and $p=0.1464 \mathrm{~m}$ to study the material properties of line-transformed cloaks. The parameter components have been illustrated in Fig. 2(b). When selecting $p=0.0476 \mathrm{~m}$, then we have $\varepsilon_{\xi}=\mu_{\xi}=0.25, \varepsilon_{\eta}=\mu_{\eta}=4$, and the $z$ components range from 0 to 4 . When $p=14.64$, then $\varepsilon_{\xi}=\mu_{\xi}=0.5, \varepsilon_{\eta}=\mu_{\eta}=2$, and the $z$ components range from 0 to 2 . In these two cases, the material properties in the cloaking region are much easier to achieve using metamaterial structures. We will investigate the cloaking performance of the line-transformed cloaks for such two cases in the next section.

\section{Discussions and simulations}

To illustrate the invisible properties of the line-transformed cloaks, we report the results of some accurately numerical simulations. All simulations are performed by using the software package, COMSOL Multiphysics, which is based on the finite element method (FEM). Here, both TE-polarized and TM-polarized time- 
harmonic incident plane waves will be considered. For the TE-wave incidence, only $\mu_{\xi}, \mu_{\eta}$, and $\varepsilon_{z}$ components of the material parameters are required for the simulations; for the TM-wave incidence, only $\varepsilon_{\xi}, \varepsilon_{\eta}$, and $\mu_{z}$ are of interest. The working frequency is chosen as $3 \mathrm{GHz}$, at which the required material properties are easily realized by artificial metamaterials. As mentioned above, in all simulations, the lengths of major axes for inner and outer elliptical shells of the cloak are set as $0.2 \mathrm{~m}$ and $0.4 \mathrm{~m}$, respectively, and the two ellipses have the common focus.

First, we consider the case when the line segment is chosen as $2 p=0.0952 \mathrm{~m}$ and TE-polarized plane waves are horizontally incident from the left to the right. Figure 3(b) shows the total electric-field distribution of the line-transformed cloak, which contains a PEC inner boundary. In Fig. 3(a), the electric-field distribution of a PEC line is shown, in which the 'dashed line' indicates the outer boundary of the cloaking layer used in the design of the line-transformed cloak. We draw this boundary only for the sake of visualization. By comparison of Figs. 3(a) and 3(b), we observe that the scattering pattern of the line-transformed cloak with PEC inner boundary is exactly the same as that of a PEC line. To illustrate this better, we calculate the scattering widthes for the bare PEC cylinder, the line-transformed cloak, and the corresponding PEC line, as plotted in Fig. 3(c). The scattering widthes and field distributions reveal that the line-transformed cloak with PEC inner boundary is perceived as a PEC line when observed anywhere outside the boundary of the cloaking layer.

When TM-polarized waves are incident along the horizontal direction, the total magnetic-field distribution of the line-transformed cloak with the PEC inner boundary is illustrated in Fig. 4(b). In such a case, the magnetic-field distribution behave exactly the same as that of a PEC line shown in Fig. 4(a). Evidently, a much smaller scattering outside the cloaking region is observed in Figs. 4(a) and 4(b). The far-field scattering patterns for the cloak and PEC line are exactly the same, as demonstrated in Fig. 4(c). Hence, a line-transformed cloak with PEC inner boundary is reshaped as a PEC line scatterer.

Next we consider the case when the PEC line is chosen as $2 p=0.2928 \mathrm{~m}$. Figure 5 illustrates the numerical results of electric fields in the computational domain under the incidence of TE-polarized plane waves. When the waves are incident along the horizontal direction, the electric-field distributions of the line-transformed cloak with the PEC inner boundary and the PEC line are shown in Figs. 5(a) and 5(b), from which we clearly see that the field values are exactly the same. The comparison of scattering widthes is shown in Fig. 5(c). When the TM-polarized plane waves are incident, the magnetic-field distributions behave significantly different from those in Fig. 5, as demonstrated in Fig. 6. Clearly, the near-filed and far-field distributions are exactly identical for the cloak and the PEC line, as shown in Figs. 6(a), 6(b) and 
$6(\mathrm{c})$.

The above simulation results verify that a line-transformed cloak with a PEC inner boundary scatters the waves exactly like a certain PEC line for both TE-wave and TM-wave incidences. In other words, the line-transformed cloak with the PEC inner boundary is indeed a reshaper of the PEC line. Hence, for the line-transformed cloak with the PEC inner boundary, the significant difference between TE-wave and TMwave incidences is similar to the case for a PEC line. From above simulation results, we also observe that the line-transformed cloak have strong scattering for the TE-wave incidence (see Figs. 3(b) and 5(b)), but have weak scattering for the TM-wave incidence (see Figs. 4(b) and 6(b)). Such a effect can be explained from the viewpoint of the cloaking shell which shrinks the PEC inner boundary to the PEC line. It is well-known that a PEC line forces the tangential electric field to zero, which can be observed in Figs. 3(a) and 5(a) for the TE incidence. However, the PEC line can support a discontinuity of the tangential magnetic field and the scattering decreases significantly for the TM incidence, as shown in Figs. 4(a) and 6(a). Hence, for the TM incident case, the cloaking performance is significantly better.

\section{Summary}

In summary, we have studied the scattering properties of a class of line-transformed cloaks numerically for both TE and TM incidences of plane waves. Such a cloak with the PEC inner boundary is indeed a reshaping of a PEC line to which the shielded object is crushed. The numerical results of near-field distributions and far-field scattering patterns have confirmed the above conclusions. We have also investigated the relationship between the material parameters of the line-transformed cloak and the length of the corresponding line, which provides an efficient way to realize the invisibility cloaks using artificial metamaterials.

\section{Acknowledgement}

This work was supported in part by the National Science Foundation of China under Grant Nos. 60990320, 60990324, 60671015, 60871016, and 60901011, in part by the Natural Science Foundation of Jiangsu Province under Grant No. BK2008031, and in part by the 111 Project under Grant No. 111-2-05. WXJ acknowledges the support from the Graduate Innovation Program of Jiangsu Province under No. CX08B_074Z. 


\section{References}

[1] J. B. Pendry, D. Schurig, and D. R. Smith, Science 312, 1780 (2006).

[2] U. Leonhardt, Science 312, 1777 (2006).

[3] D. Schurig, J. J. Mock, B. J. Justice, S. A. Cummer, J. B. Pendry, A. F. Starr, and D. R. Smith, Science 314, 977 (2006).

[4] J. Li and J. B. Pendry, Phys. Rev. Lett. 101, 203901 (2008).

[5] R. Liu, C. Ji, J. J. Mock, J. Y. Chin, T. J. Cui, D. R. Smith, Science 323, 366 (2009).

[6] L. H. Gabrielli, J. Cardenas, C. B. Poitras and M. Lipson, Nat. Photon. 3, 461 (2009).

[7] J. Valentine, J. Li, T. Zentgraf, G. Bartal, and X. Zhang, Nat. Mater. 8, 568 (2009).

[8] D. Schurig, J. B. Pendry, and D. R. Smith, Opt. Express 14, 9794 (2006).

[9] S. A. Cummer, B.-I. Popa, D. Schurig, D. R. Smith, and J. B. Pendry, Phys. Rev. E 74, 036621 (2006).

[10] A. Hendi, J. Henn, and U. Leonhardt, Phys. Rev. Lett. 97, 073902 (2006).

[11] D. A. B. Miller, Opt. Express 14, 12457 (2006).

[12] A. Alu and N. Engheta, Phys. Rev. Lett. 100, 113901 (2008).

[13] W. X. Jiang, J. Y. Chin, Z. Li, Q. Cheng, R. Liu, and T. J. Cui, Phys. Rev. E 77, 066607 (2008).

[14] Z. Ruan, M. Yan, C. W. Neff, and M. Qiu, Phys. Rev. Lett. 99, 113903 (2007).

[15] W. Cai, U. K. Chettiar, A. V. Kildishev, and V. M. Shalaev, Nature Phot. 1, 224 (2007).

[16] H. Chen, B. I. Wu, B. Zhang, and J. A. Kong, Phys. Rev. Lett. 99, 063903 (2007).

[17] D.-H. Kwon and D.H. Werner, Appl. Phys. Lett. 92, 013505 (2008).

[18] H. Ma, S. Qu, Z. Xu, J. Zhang, B. Chen, and J. Wang, Phys. Rev. A 77, 013825 (2008).

[19] D.-H. Kwon and D.H. Werner, Appl. Phys. Lett. 92, 113502 (2008).

[20] W. X. Jiang, T. J. Cui, G. X. Yu, X. Q. Lin, Q. Cheng, and J. Y. Chin, J Phys. D: Appl. Phys. 41, $085504(2008)$.

[21] Y. You, G.W. Kattawar, P.W. Zhai, and P. Yang, Opt. Express 16, 6134 (2008). 
[22] W. X. Jiang, T. J. Cui, X. M. Yang, Q. Cheng, R. Liu, and D. R. Smith, Appl. Phys. Lett. 93, 194102 (2008).

[23] W. Yan, M. Yan, Z. Ruan and M. Qiu, New J. Phys. 10, 043040 (2008). 


\section{List of Figure Captions}

Fig. 1: (color online) A scheme of the cloak's inner boundary for (a) a point-transformed cloak and (b) a line-transformed cloak. (c) The cross section of a general cylindrical cloak (green region).

Fig. 2: (color online) (a) Permittivity and permeability components for the line-transformed cloak when the line segment $2 p$ changes from 0 to $2 r_{1}$. (b) Permittivity and permeability components for two different cases when $p=0.0476 \mathrm{~m}$ and $p=0.1464 \mathrm{~m}$.

Fig. 3: (color online) TE-polarized plane waves are incident along the horizontal direction when $p=0.0476$ m. The electric-field distributions of (a) the line-transformed invisibility cloak with the PEC inner boundary, (b) the PEC line. (c) The scattering width for the line-transformed cloak, the PEC line and a bare PEC cylinder.

Fig. 4: (color online) TM-polarized plane waves are incident horizontally when $p=0.0476 \mathrm{~m}$. The magnetic-field distributions of (a) the line-transformed invisibility cloak with the PEC inner boundary, (b) the PEC line. (c) The scattering width for the line-transformed cloak, the PEC wire, and the bare PEC cylinder.

Fig. 5: (color online) TE-polarized plane waves are incident along the horizontal direction when $p=0.1464$ m. The electric-field distributions of (a) the line-transformed invisibility cloak with the PEC inner boundary, (b) the PEC line. (c) The scattering width for the line-transformed cloak, the PEC line, and the bare PEC cylinder.

Fig. 6: (color online) TM-polarized plane waves are incident horizontally when $p=0.1464 \mathrm{~m}$. The magnetic-field distributions of (a) the line-transformed invisibility cloak with the PEC inner boundary, (b) the PEC line. (c) The scattering width for the line-transformed cloak, the PEC line, and the bare PEC cylinder. 


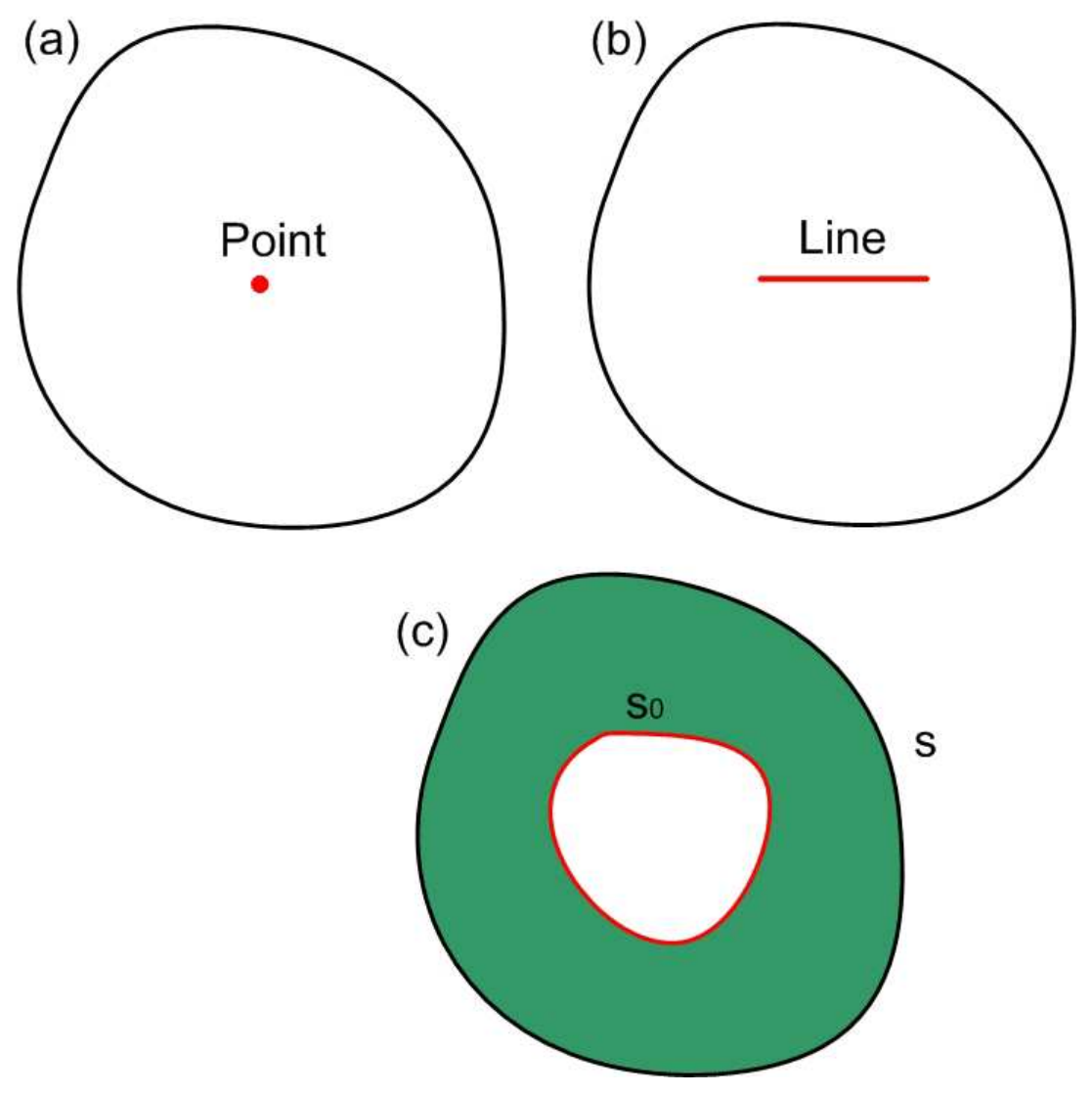

Figure 1: 
(a)

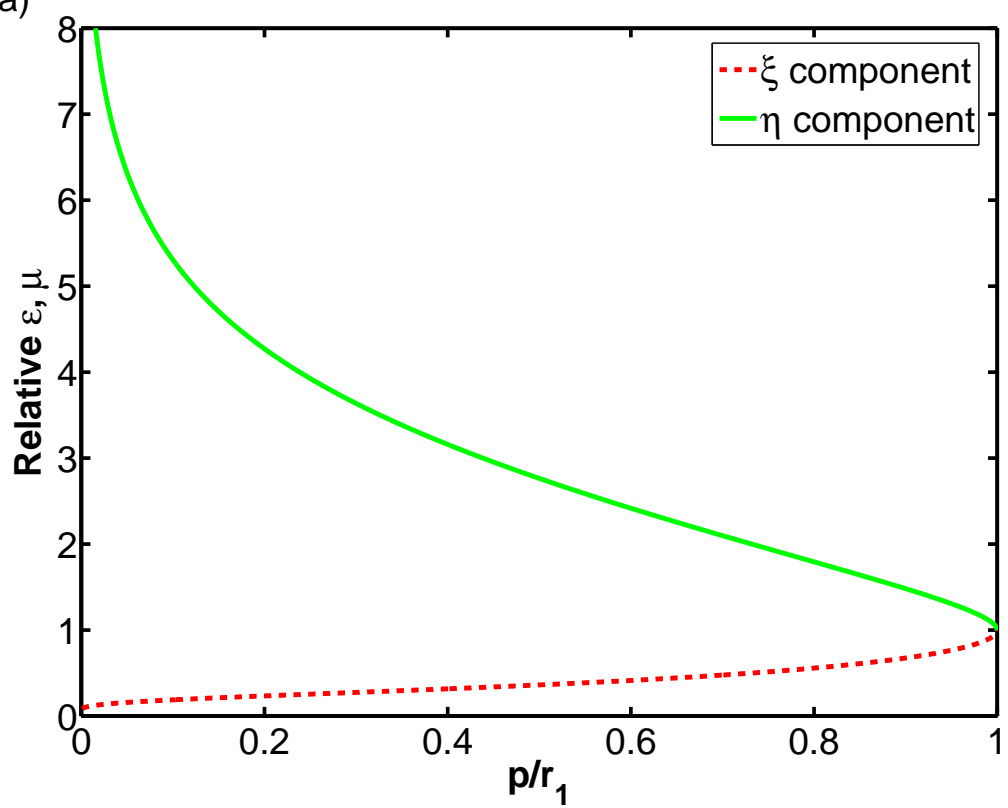

(b)

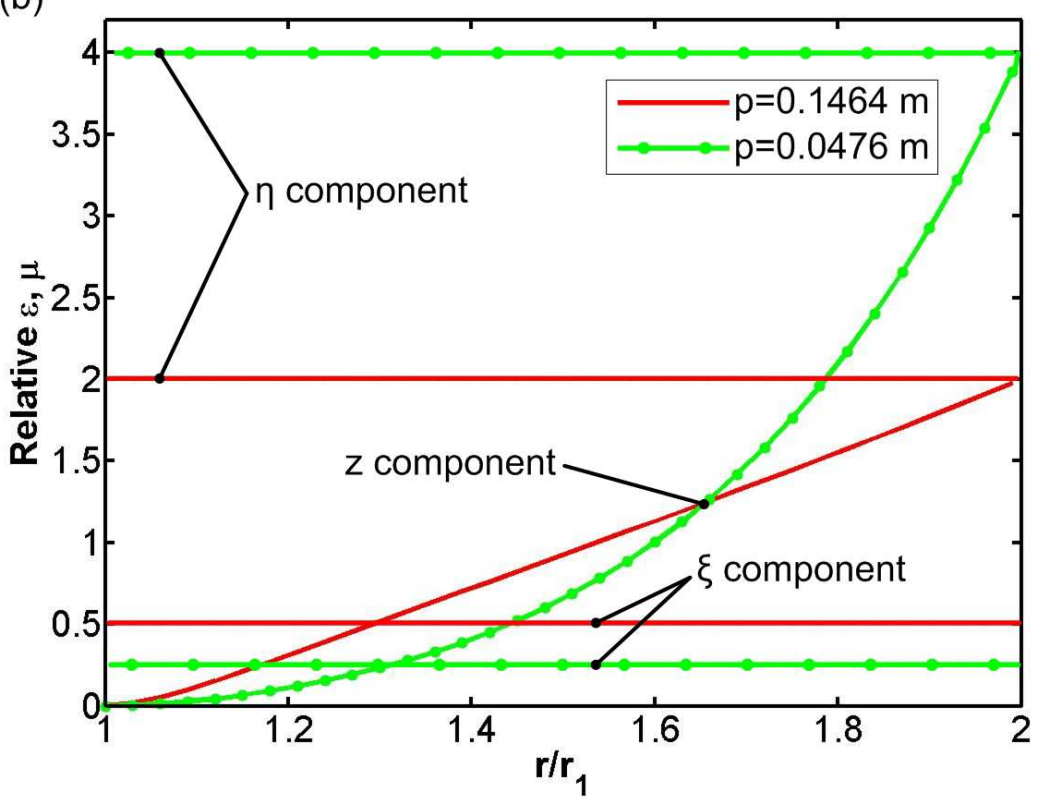

Figure 2: 

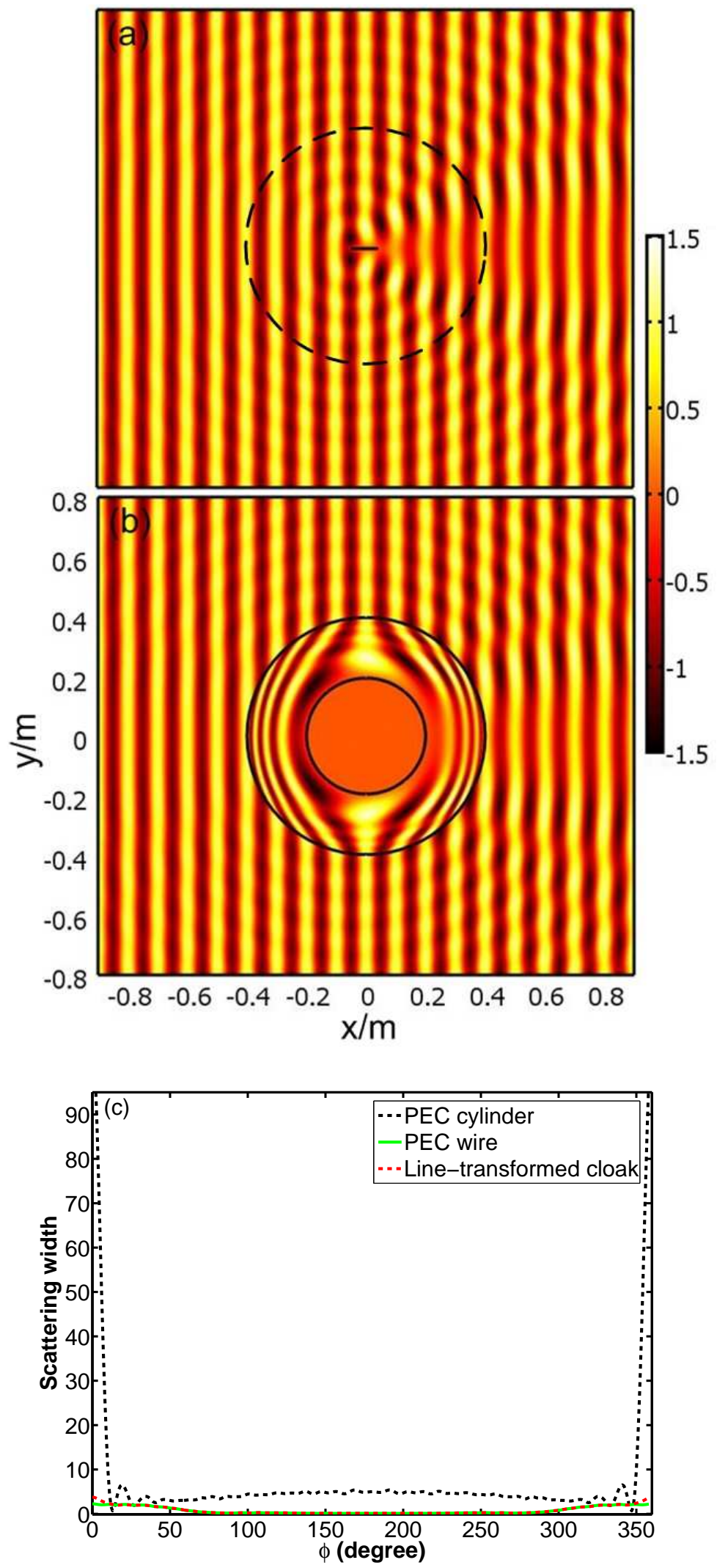

Figure 3: 

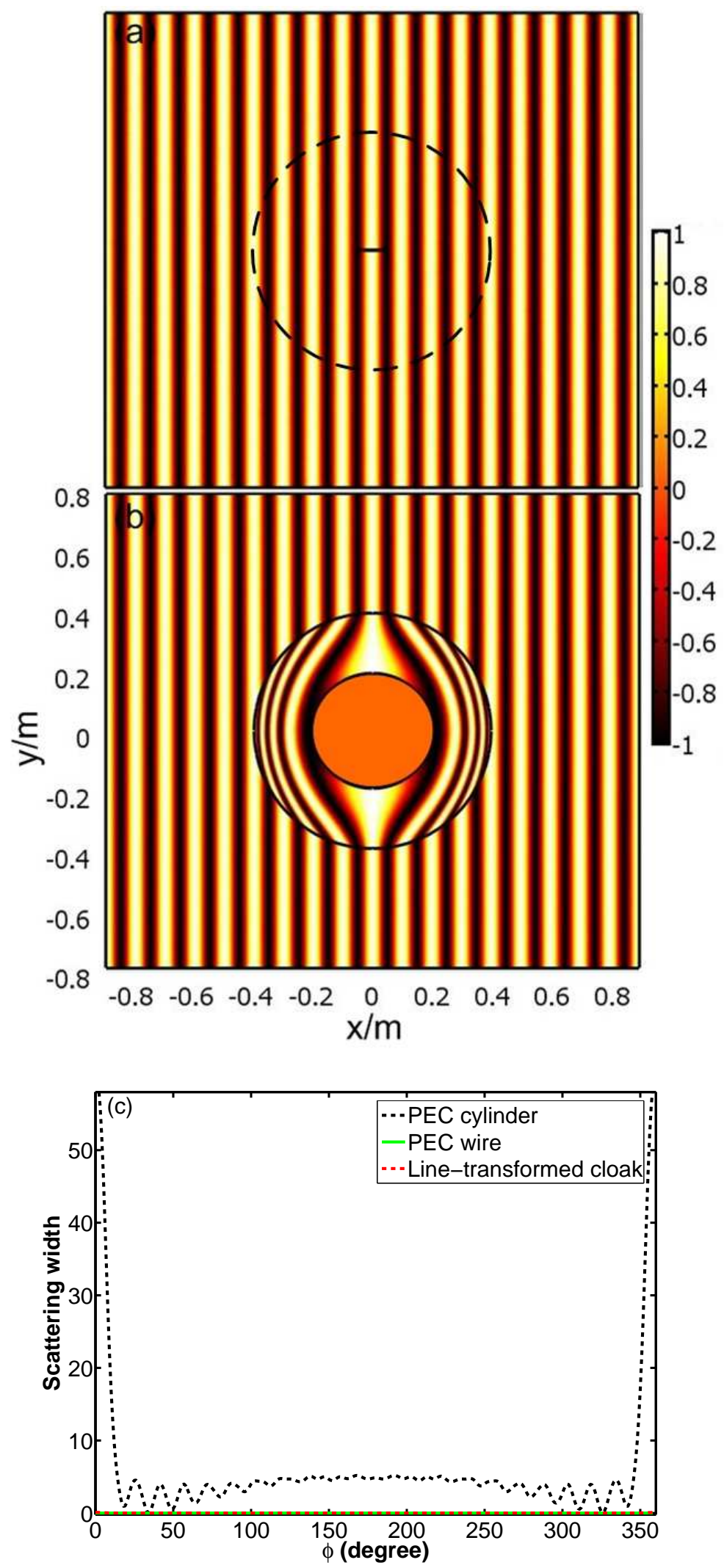

Figure 4: 

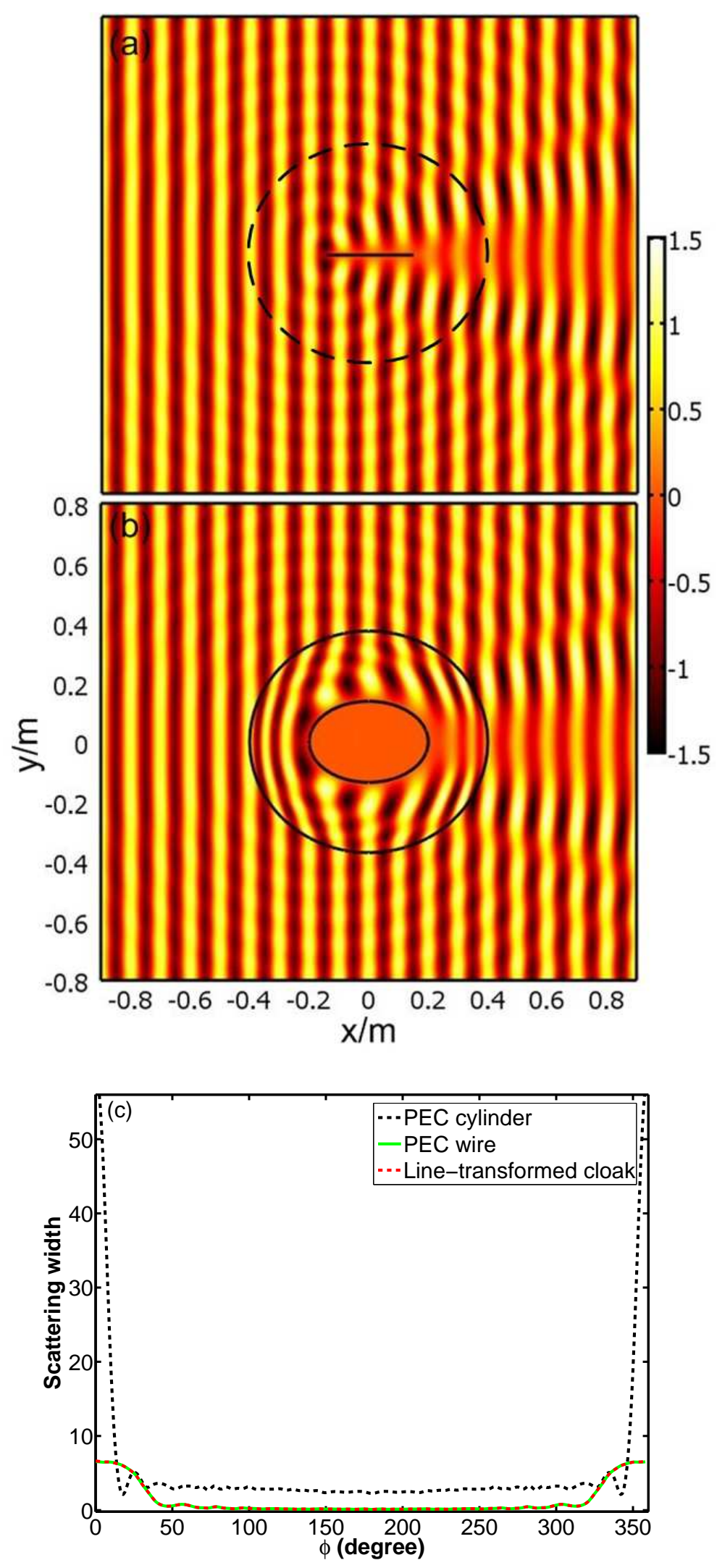

Figure 5: 

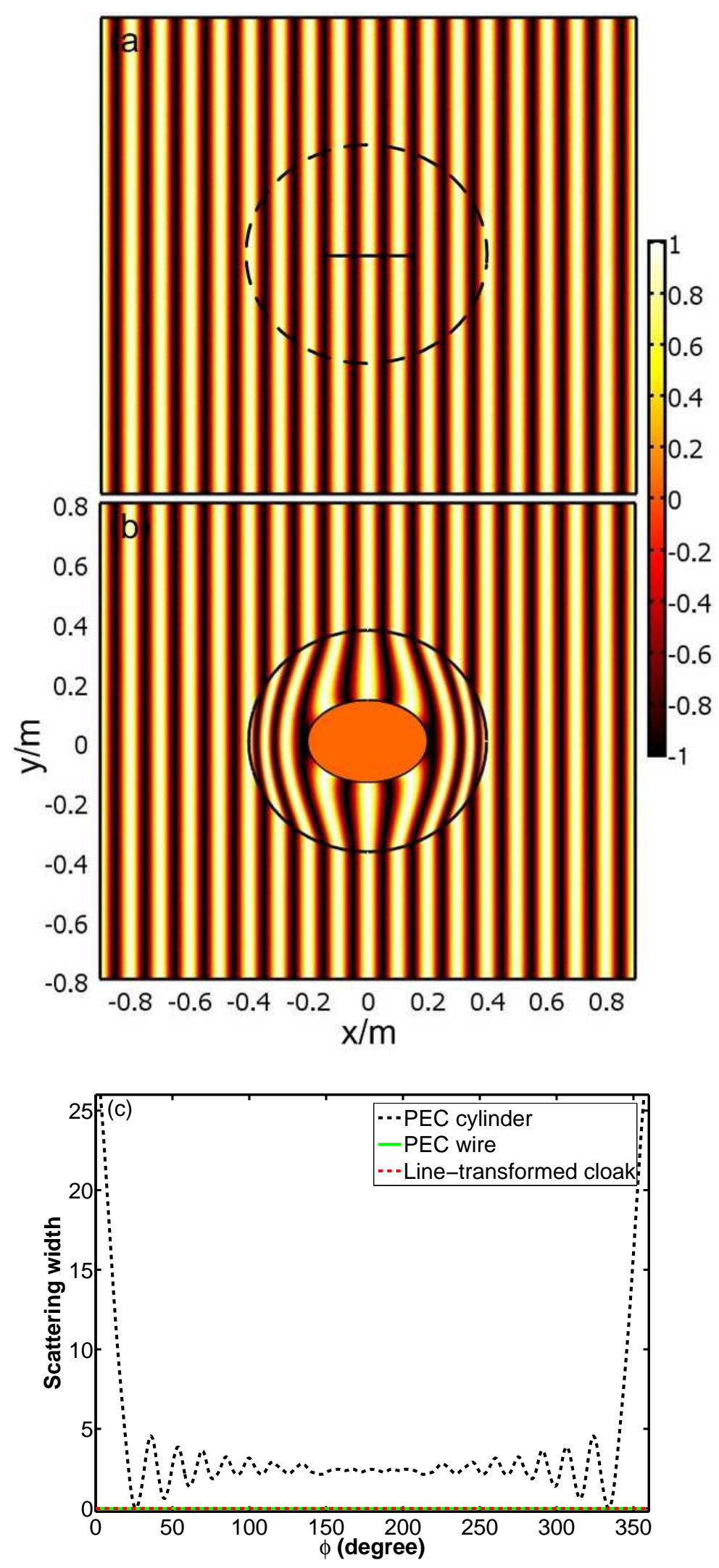

Figure 6: 